\title{
A!
}

This is an electronic reprint of the original article.

This reprint may differ from the original in pagination and typographic detail.

Vehviläinen, T.T.; Ganchenkova, M.G.; Nieminen, R.M.

\section{Nanoporous carbon structures based on C20}

Published in:

Physical Review B

DOI:

10.1103/PhysRevB.84.125444

Published: 01/01/2011

Document Version

Publisher's PDF, also known as Version of record

Please cite the original version:

Vehviläinen, T. T., Ganchenkova, M. G., \& Nieminen, R. M. (2011). Nanoporous carbon structures based on C20. Physical Review B, 84(12), 1-7. [125444]. https://doi.org/10.1103/PhysRevB.84.125444

This material is protected by copyright and other intellectual property rights, and duplication or sale of all or part of any of the repository collections is not permitted, except that material may be duplicated by you for your research use or educational purposes in electronic or print form. You must obtain permission for any other use. Electronic or print copies may not be offered, whether for sale or otherwise to anyone who is not an authorised user. 


\title{
Nanoporous carbon structures based on $\mathbf{C}_{20}$
}

\author{
T. T. Vehviläinen, ${ }^{*}$ M. G. Ganchenkova, and R. M. Nieminen \\ COMP/Department of Applied Physics, School of Science, Aalto University, P.O. Box 11100, FI-00076 Aalto, Espoo, Finland
}

(Received 28 February 2011; revised manuscript received 29 June 2011; published 28 September 2011)

\begin{abstract}
In this paper, we present computational results for $\mathrm{C}_{20}$ based solids. We propose structures that are shown to be energetically more favorable and stable than previously suggested structures. The so-called quasigraphite phase and base-centered-monoclinic type structures are found to be the energetically most favorable. The moleculardynamics stability of suggested structures was studied via constant-temperature and constant-pressure techniques and by examining phonon dispersion curves. All the predicted structures demonstrate high stability with respect to temperature and external load. By changing the geometry, the electronic properties can be varied from metallic to insulating.
\end{abstract}

DOI: 10.1103/PhysRevB.84.125444

PACS number(s): 81.05.U-, 78.30.Na, 62.20.-x, 31.15.A-

\section{INTRODUCTION}

Porous carbon structures, especially zeolite-like carbon materials have been shown to have very high hydrogen storage capacity, which is reversible up to $8.33 \mathrm{wt} \% .{ }^{1}$ This uptake capacity is one of the highest reported for carbon nanomaterials, and therefore makes similar porous carbon structures promising for hydrogen storage applications. In addition to hydrogen storage, ${ }^{2-4}$ fullerene-based structures are possible candidates for superconductors, ${ }^{5}$ strong lightweight materials, quantum dots, ${ }^{6,7}$ and biological applications. ${ }^{8,9}$

It has been shown that zeolites, which are aluminosilicate minerals and have a microporous structure, can be used as templates for nanostructures where carbon fills the pores and forms three-dimensional networks. ${ }^{10,11}$ After the synthesis of the carbon nanostructure, the zeolite template is removed by washing the structure using hydrofluoric acid. ${ }^{1,11}$ The resulting material is expected to have pores in original template areas and carbon networks inside original pores, resulting in structural pore ordering similar to that of the zeolite. ${ }^{1}$ The solids formed when fullerene molecules ${ }^{12}$ form three-dimensional (3D) lattices, have similar porous geometry compared to structures synthetized using zeolite templates. Therefore fullerene solids can serve as excellent model systems for nanotemplated porous carbon nanostructures synthesised in experiments.

So far, mostly $\mathrm{C}_{60}$-based polymers and solids have been studied, both experimentally and theoretically. ${ }^{13,14}$ However, the recent synthesis of a broad variety of fullerene cages has opened perspectives to the creation of other kinds of solid structures with different fullerenes as building blocks. We have chosen the smallest fullerene $\mathrm{C}_{20}$ as a building block for solid structures. This fullerene consists of 12 pentagons and no hexagons, while in other fullerenes there are also hexagons in the structure. The extreme curvature of the cage surface makes the dihedral angles between bonds $\left(108^{\circ}\right)$ more appropriate to $s p^{3}$ hybridization $\left(109.5^{\circ}\right)$ than $s p^{2}$, although the $\mathrm{C}_{20}$ molecule exhibits $s p^{2}$ hybridization. Therefore it can be expected that $\mathrm{C}_{20}$ changes hybridization type especially easily, which is a prerequisite for chemical bonding between fullerenes during polymerization process and should manifest extreme reactivity and easy compound formation. However, so far, there have been only two succesfull attempts where three-dimensional $\mathrm{C}_{20}$-based solid has been experimentally produced. ${ }^{15-17}$ In Ref. 15, based on the comparison of computational and experimental Raman and IR spectra, Iqbal et al. have shown that the best fit to the experimental data is demonstrated by a face-centered cubic $\mathrm{C}_{22}$ structure, where $\mathrm{C}_{20}$ molecules are located on face-centered cubic lattice sites and are bound by two additional $\mathrm{C}$ atoms. Whereas Wang et al. ${ }^{17}$ managed to synthesize a small amount of hexagonal $\mathrm{C}_{20}$ solid using ion beam irradiation method. According to computational studies, other stable 3D solid structures might exist, including simple cubic (sc), ${ }^{5,18,19}$ face-centered cubic (fcc), ${ }^{18,19}$ and body-centered cubic (bcc) ${ }^{18-20}$ phases.

In this article, we propose, based on density-functionaltheory calculations, a number of stable $\mathrm{C}_{20}$-based structures. Among these structures are both those described earlier ${ }^{5,18,20}$ and those which to our knowledge have not been considered before. Moreover, the structures proposed in this work are energetically the most favorable and stable as compared to previously suggested ones. ${ }^{5,18,20}$ The electronic structure, stability, and elastic and vibrational properties of the considered structures are also reported in this paper.

\section{COMPUTATIONAL DETAILS}

The calculations have been performed using the planewave basis VASP code ${ }^{21,22}$ implementing the spin-polarized density-functional theory (DFT) and the generalized gradient approximation (GGA) of Perdew and Wang known as PW91. ${ }^{23}$ In order to describe the carbon ion core electrons, the projector augmented wave (PAW) potential ${ }^{24,25}$ was used. Periodic boundary conditions were used in order to represent $C_{20}$ solids. A kinetic energy cutoff of $400 \mathrm{eV}$ and $6 \times 6 \times 6$ Brillouin-zone sampling were used. These values provide the convergence of the supercell total energy within $10 \mathrm{meV}$. The relaxations of atomic positions and lattice parameters were performed with molecular-statics methods and the conjugate-gradient algorithm. In order to reproduce different $\mathrm{C}_{20}$ structures, the supercell shape was adjusted so that the cell repetition due to periodic boundary conditions would provide the desired overall lattice symmetry. The supercell size was chosen initially so large that the intermolecular separation would suffice to prevent the interaction between the molecule and its periodic images. Then the supercell was gradually decreased until the molecules bonded to each other, forming two- or three-dimensional structures. After the first energy minimum was reached, the cell compression was further 
continued in order to study the possible pressure-induced phase transformations of the structure. For this purpose, both isotropic and anisotropic compressions were used. In this way, we have systematically studied the most reasonable structures, which can be created using a 20-atom supercell.

For molecular dynamics simulations and stability studies we used the $\mathrm{CPMD}^{26,27}$ code, where the Parrinello-Rahman constant-pressure constant-temperature technique and the DFT-GGA approximation are implemented. The size of the supercell for stability studies was 160 atoms and the Brillouinzone sampling was done with the $\Gamma$ point.

Phonon-related properties were calculated using the ABINIT $^{28}$ code with the local-density approximation (LDA) and density-functional perturbation theory with a $4 \times 4 \times 4$ grid for special high-symmetry $q$ points and a $680-\mathrm{eV}$ kinetic energy cutoff for plane waves.

Some of the studied solids have graphitelike layered structure. In this case, the account of van der Waals (vdW) interactions becomes crucial. In order to take the vdW interaction into account, we have used the empirical damped dispersion model, as described by M. Elstner et al. ${ }^{29}$ and having the same form as constructed by Mooij et al ${ }^{30,31}$ This empirical model allows the estimation of the vdW energy, which is added to the standard DFT energy to obtain total energy of the electron-ion system

$$
E_{\mathrm{vdW}}=\sum_{i j} \frac{C_{6}^{\alpha \beta}}{R_{i j}^{\alpha \beta^{6}}}\left\{1-\exp \left[-d\left(\frac{R_{i j}^{\alpha \beta}}{R_{0}^{\alpha \beta}}\right)^{7}\right]\right\}^{4}
$$

In this formula, $C_{6}^{\alpha \beta}$ is the diatomic vdW force coefficient, $R_{0}^{\alpha \beta}$ is the sum of vdW radii of two interacting atoms, $R_{i j}^{\alpha \beta}$ is the distance between atoms and $d$ is the damping coefficient. The total energy of the electron-ion system is $E_{\text {total }}=E_{\mathrm{DFT}}+E_{\mathrm{vdW}}$. The model ensures that bonded interactions are not doubly counted, because the vdW correction drops to zero when the distance between atoms is less than $3 \AA$. In the current calculations, we used the set of model parameters obtained by Williams and Malhotra ${ }^{32}$ using PBE and BLYP functionals and the $\mathrm{vdW}$ corection is used to determine layer-layer distances, bulk moduli and elastic constants only for layered materials. Based on our calculations, neglecting vdW corrections for the other 3D structures does not have a significant effect on the results.

The applicability of the method was tested for the graphite interplane distance optimization. The obtained results show that the measured interplane distances and those calculated with vdW correction, i.e., 3.35 and $3.1 \AA$, respectively, are in reasonable agreement. The calculated $c_{33}$ elastic constant value of $49 \mathrm{GPa}$ for graphite also reproduces reasonably well the known experimental value of $36 \mathrm{GPa} .{ }^{33}$ Comparing to the van der Waals density functional calculations, ${ }^{34}$ which give $3.76 \AA$ for the interplane distance and $13 \mathrm{GPa}$ for $c_{33}$ elastic constant, empirical model used in this study seems to give better agreement with experimental values for both lattice and elastic constants.

TABLE I. Energies and lattice parameters for studied polymers with well-known carbon structures. Energies are given without vdW energy taken into account.

\begin{tabular}{|c|c|c|c|c|c|}
\hline Structure & $\begin{array}{c}\text { E/atom } \\
(\mathrm{eV})\end{array}$ & $\begin{array}{c}\text { E/atom }{ }^{18} \\
(\mathrm{eV})\end{array}$ & El. prop. & $\begin{array}{l}\text { Lattice } \\
\text { vectors }\end{array}$ & $\begin{array}{c}\text { Bond } \\
\text { lengths }(\AA)\end{array}$ \\
\hline C-atom & -1.270 & $\ldots$ & & & $\ldots$ \\
\hline Diamond & -9.10 & -9.22 & $\mathrm{I}, E_{\mathrm{gap}}=5.6 \mathrm{eV}$ & $a=3.573 \AA$ & 1.54 \\
\hline Graphite & -9.25 & -9.24 & $\mathrm{M}$ & $a=b=2.46 \AA ̊, c=6.2 \AA$ & 1.42 \\
\hline $\mathrm{C}_{20}$ molecule & -8.07 & -8.01 & $\ldots$ & - & 1.45 \\
\hline $\mathrm{C}_{20} \mathrm{sc}_{\text {open }}$ & -8.49 & -8.48 & M & $a=5.45 \AA$ & $1.42,1.46$ \\
\hline $\mathrm{C}_{20} \mathrm{sc}_{\text {closed }}$ & -8.36 & $\ldots$ & $\mathrm{S}, E_{\text {gap }}=1.0 \mathrm{eV}$ & $\begin{array}{l}a=6.06 \AA, b=6.48 \AA \\
c=6.48 \AA\end{array}$ & $1.38,1.42,1.33,1.48$ \\
\hline $\mathrm{C}_{20} \mathrm{bcm}_{\text {closed }}$ & -8.56 & -8.56 & $\mathrm{~S}, E_{\text {gap }}=1.4 \mathrm{eV}$ & $\begin{array}{l}a_{1}=(4.28,3.81,-0.07) \AA \\
a_{2}=(-0.07,3.81,4.28) \AA \\
a_{3}=(4.21,0.00,4.22) \AA\end{array}$ & $\begin{array}{l}1.29,1.44,1.50,1.54 \\
1.61,1.62,1.64,1.69\end{array}$ \\
\hline $\mathrm{C}_{20} \mathrm{bcm}_{\text {open }}$ & -8.79 & $\ldots$ & $\mathrm{S}, E_{\text {gap }}=1.5 \mathrm{eV}$ & $\begin{array}{l}a_{1}=(4.31,4.54,0.32) \AA \\
a_{2}=(0.32,4.54,4.31) \AA \\
a_{3}=(3.98,0.65,3.98) \AA\end{array}$ & $\begin{array}{c}1.34,1.50,1.53,1.54 \\
1.56,1.57\end{array}$ \\
\hline $\mathrm{C}_{20} \mathrm{bcc}$ & -8.51 & $\ldots$ & $\mathrm{S}, E_{\text {gap }}=2.32 \mathrm{eV}$ & $\begin{array}{l}a_{1}=(-3.37,3.37,3.37) \AA \\
a_{2}=(3.37,-3.37,3.37) \AA \\
a_{3}=(3.37,3.37,-3.37) \AA\end{array}$ & $1.34,1.54$ \\
\hline $\mathrm{C}_{20}$ hcp & -8.49 & $\ldots$ & M & $\begin{array}{l}a_{1}=(2.86,5.57,0.00) \AA \\
a_{2}=(2.86-5.57,0.00) \AA \\
a_{3}=(0.00,0.00,6.2) \AA\end{array}$ & $\begin{array}{c}1.34,1.39,1.41 \\
1.47,1.50\end{array}$ \\
\hline $\mathrm{C}_{20}$ qgp & -8.91 & $\cdots$ & M & $\begin{array}{l}a=4.91 \AA, b=5.36 \AA \\
c=6.0 \AA\end{array}$ & $1.41,1.46,1.51,1.55$ \\
\hline $\mathrm{C}_{22} \mathrm{fcc}$ & -8.56 & $\ldots$ & $\mathrm{S}, E_{\text {gap }}=2.57 \mathrm{eV}$ & $\begin{array}{l}a_{1}=(0.00,4.38,4.38) \AA \\
a_{2}=(4.38,0.00,4.38) \AA \\
a_{3}=(4.38,4.38,0.00) \AA\end{array}$ & $1.35,1.54,1.56$ \\
\hline
\end{tabular}




\section{RESULTS AND DISCUSSION}

\section{A. Atomic structure}

We have systematically created various phases of $\mathrm{C}_{20}$ solids as described in Sec. II. First, we focus on base-centered monoclinic (bcm) structures, which are found to be energetically the most favorable ones among typical lattice symmetries, see Table I. Different bcm structures can be created using initial bcm symmetry by variation of mutual orientation of $\mathrm{C}_{20}$ molecules constituting the lattice and applying different strains to the structure. In this paper we present two $\mathrm{C}_{20} \mathrm{bcm}$ solid structures (see Fig. 1), which are called here bcm $_{\text {open }}$ and

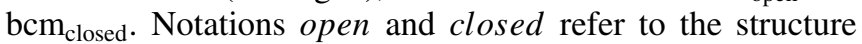
of the fullerene cage after the polymerization process. In the open structure bonds are broken, opening the cage, while in the closed structure there is only bond formation and all bonds in the original fullerene remain intact. The $\mathrm{bcm}_{\text {closed }}$ structure has been proposed earlier by Okada. ${ }^{20}$ However, another bcm-type structure, predicted in this work, turned out to be pronouncedly more favorable, being characterized by the energy of $-8.79 \mathrm{eV} /$ atom, as compared to -8.56 $\mathrm{eV} /$ atom for $\mathrm{bcm}_{\text {closed }}{ }^{20}$ In the $\mathrm{bcm}_{\text {closed }}$ structure, almost all carbon atoms are $s p^{3}$ hybridized, while only four atoms remain $s p^{2}$ hybridized. All the bonds of the fullerene cage remain unbroken and the cage is closed. However, this is not the case for the $\mathrm{bcm}_{\text {open }}$ structure, where two bonds of the fullerene cage are broken, which leads to the formation of four new bonds between neighboring molecules and to cage opening. Each carbon atom of the fullerene cage takes part in the bonding. Carbon atoms with broken bonds form new bonds with neighboring fullerenes and remain $s p^{2}$ hybridized, while the others become $s p^{3}$ hybridized during the polymerization process. Also, as can be seen in Fig. 1, the cage constituting the $\mathrm{bcm}_{\text {open }}$ solid structure is slightly inclined compared to the $\mathrm{bcm}_{\text {closed }}$ structure.

The $\mathrm{C}_{22}$ fcc structure, which we have reproduced in our studies, is also one of the most interesting structures since it is claimed to be synthetized in experiments. ${ }^{15,16}$ In this structure, the $\mathrm{C}_{20}$ cages are interlinked together by two interstitial carbon
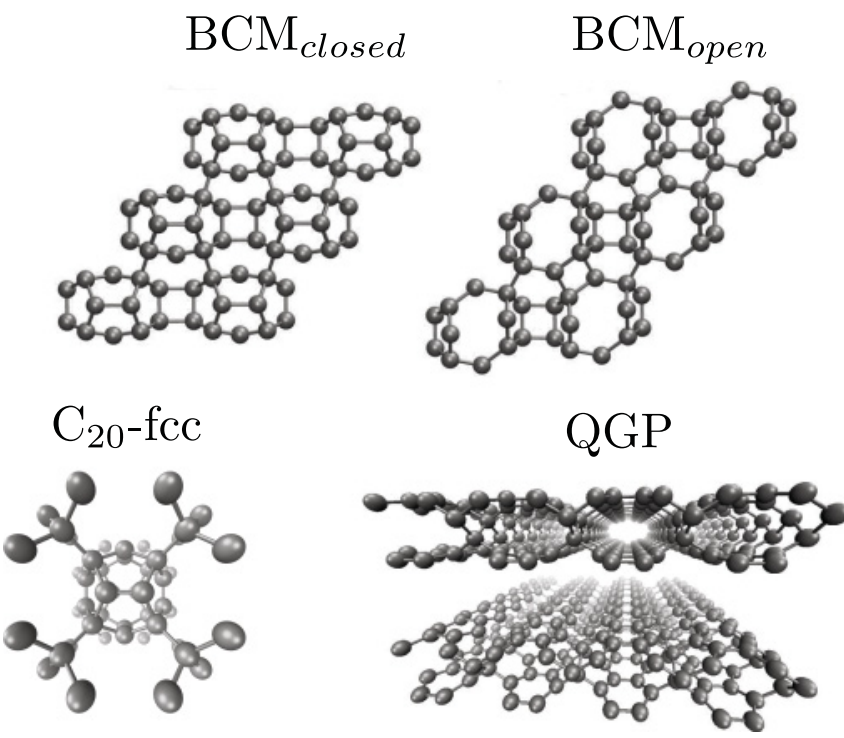

FIG. 1. Geometry of $\mathrm{C}_{20}$ bcm-type, qgp, and $\mathrm{C}_{22}$-fcc structures.
TABLE II. Elastic properties of studied polymers. $\mathrm{B}^{a}$ is bulk modulus calculated using Murnaghan equation of state $E(V)=$ $B^{a} V /\left[B^{a^{\prime}}\left(B^{a^{\prime}}-1\right)\right]\left[B^{a^{\prime}}\left(1-V_{0} / V\right)+\left(V_{0} / V\right)^{B^{a^{\prime}}}-1\right], \mathrm{B}^{b}$ is bulk modulus calculated using elastic constants $B^{b}=1 / 9\left(c_{11}+c_{22}+\right.$ $\left.c_{33}\right)+2 / 9\left(c_{13}+c_{23}+c_{13}\right)$.

\begin{tabular}{lccccccccc}
\hline \hline Structure & $\mathrm{c}_{11}$ & $\mathrm{c}_{22}$ & $\mathrm{c}_{33}$ & $\mathrm{c}_{44}$ & $\mathrm{c}_{66}$ & $\mathrm{c}_{12}$ & $\mathrm{c}_{23}$ & $\mathrm{~B}^{a}$ & $\mathrm{~B}^{b}$ \\
\hline Diamond & 1085 & $\ldots$ & $\ldots$ & 569 & $\ldots$ & 140 & $\ldots$ & 447 & 455 \\
Graphite & 1058 & $\ldots$ & 49 & $\ldots$ & $\ldots$ & $\ldots$ & $\ldots$ & 34 & $\ldots$ \\
$\mathrm{C}_{20} \mathrm{sc}_{\text {open }}$ & 468 & $\ldots$ & $\ldots$ & 41 & $\ldots$ & 134 & $\ldots$ & 247 & 245 \\
$\mathrm{C}_{20} \mathrm{sc}_{\text {closed }}$ & 221 & 607 & $20-30$ & $\ldots$ & 67 & 110 & $\ldots$ & $15-20$ & $\ldots$ \\
$\mathrm{C}_{20}$ bcm $_{\text {closed }}$ & 723 & $\ldots$ & 581 & 207 & 304 & 108 & 179 & 286 & 312 \\
$\mathrm{C}_{20}$ bcm $_{\text {open }}$ & 753 & $\ldots$ & 863 & 324 & 431 & 111 & 211 & 345 & 359 \\
$\mathrm{C}_{20}$ bcc & 375 & $\ldots$ & $\ldots$ & 165 & $\ldots$ & 106 & $\ldots$ & 190 & 195 \\
$\mathrm{C}_{20}$ hcp & 633 & 300 & $20-35$ & $\ldots$ & 17 & 49 & $\ldots$ & $20-30$ & $\ldots$ \\
$\mathrm{C}_{20}$ qgp & 1050 & 636 & 48 & $\ldots$ & 340 & 90 & $\ldots$ & 50 & $\ldots$ \\
$\mathrm{C}_{22} \mathrm{fcc}$ & 447 & $\ldots$ & $\ldots$ & 218 & $\ldots$ & 109 & $\ldots$ & 214 & 221 \\
\hline \hline
\end{tabular}

atoms per unit cell. These interstitial carbon atoms and eight atoms in the fullerene cage are $s p^{3}$ hybridized while 12 atoms remain $s p^{2}$ hybridized. The calculated bond lengths are in agreement with $1.346,1.517$, and $1.531 \AA$ of Iqbal et al., see Table I.

Under isotropic compression a phase transformation in the $\mathrm{sc}_{\text {closed }}$ (see Fig. 2) polymer structure was found to take place when the pressure reached the value of $30 \mathrm{GPa}$. According to the general scheme described above, the fullerene cages are initially packed in a simple cubic lattice, facing each other with parallel edges. Gradual isotropic compression of this system eventually results in the equilibrium layered $\mathrm{sc}_{\text {closed }}$ structure. If, however, the hydrostatic compression is continued, another phase transformation takes place and a new high-pressure phase, the quasigraphite phase qgp, ${ }^{35}$ is formed. The structure of the qgp can be described as an arrangement of small

$$
\mathrm{SC}_{\text {closed }}
$$
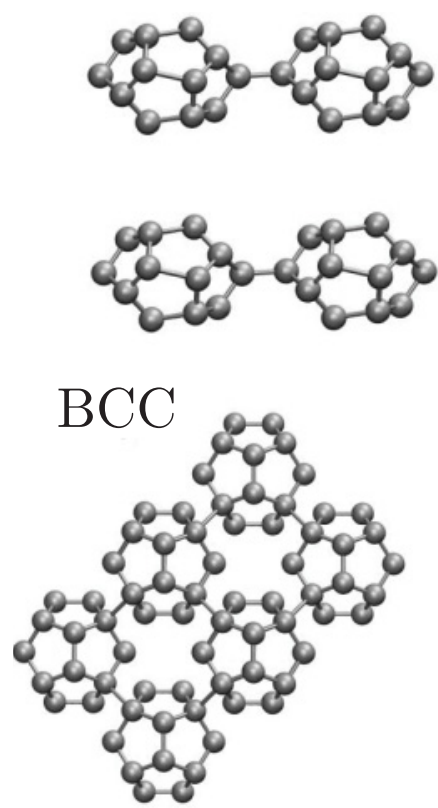

$\mathrm{SC}_{\text {open }}$

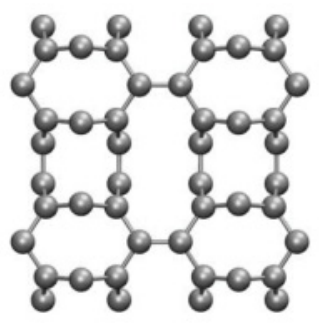

$\mathrm{HCP}$

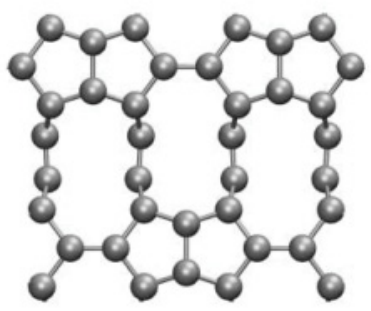

FIG. 2. Geometry of $\mathrm{C}_{20}$ sc-type, bcc, and hcp structures. 
nanotubes welded together forming planar layers, which are stacked in a graphitelike manner (see Fig. 1). Based on the surface geometry of the tubes, the qgp can be regarded as armchair structure. Due to the welding, tubes are not circular but rather ellipsoids where the minor and major radii are $a=3.0 \AA$ and $b=5.4 \AA$, respectively. The atoms that provide nanotube welding manifest the $s p^{3}$ hybridization, while other atoms constituting nanotubes have $s p^{2}$ hybridization. The distance between the qgp planes is also similar to that in graphite and equals to $3.0 \AA$. The energy of the qgp structure is $-8.91 \mathrm{eV} /$ atom, which is not only more favorable than the intermediate sc structure, but turns out to show the largest cohesive energy among all the equilibrium 3D solids based on the $\mathrm{C}_{20}$ fullerene (see Table I). In our procedure of creating solids, the transition is induced exclusively by application of pressure, without any assistance of temperature since the temperature is always zero for the molecular-statics relaxation used here.

The least favorable structures are bcc and hcp structures with energies $-8.51 \mathrm{eV}$ and $-8.49 \mathrm{eV}$, respectively. During

(a)
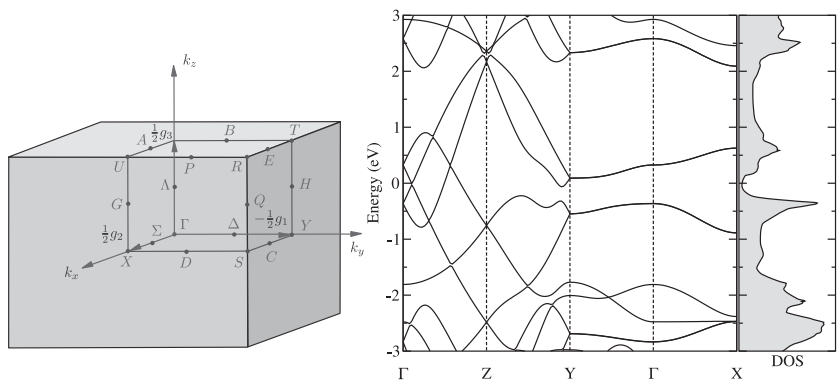

(c)
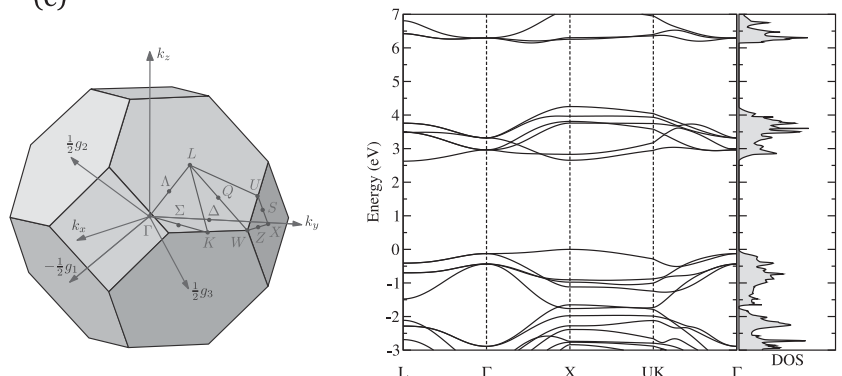

(d)

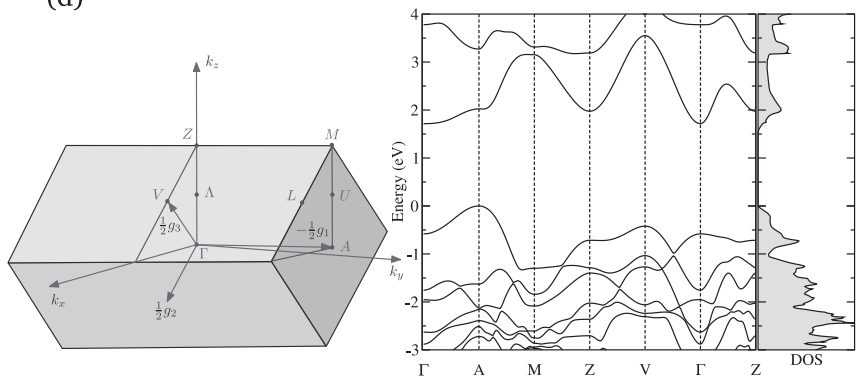

(e)
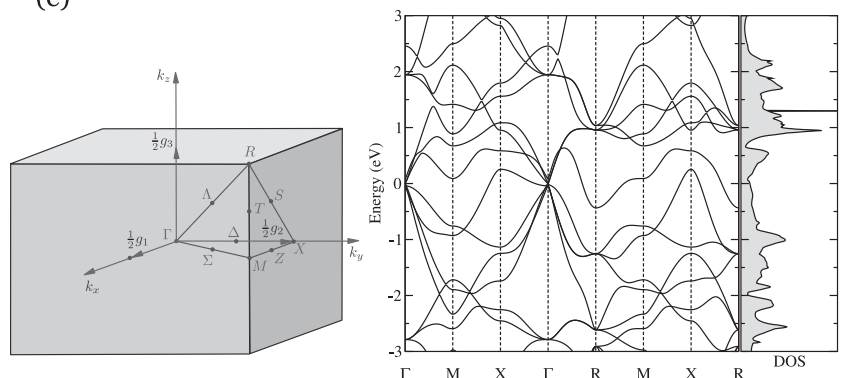

(f)

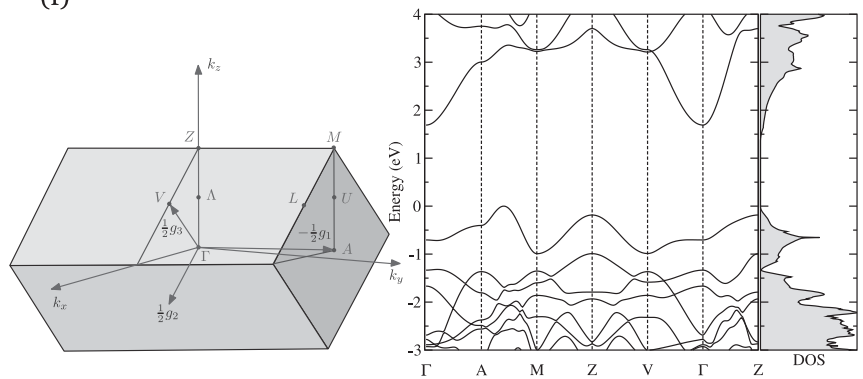

FIG. 3. Band structure plots for energetically most favorable structures: (a) qgp, (b) bcc, (c) fcc-22, (d) bcm open, (e) sc, and (f) bcm closed 
results show, in particular, that elastic constants for the fcc structures are large and the structures are, therefore, very rigid, which correlates with the large number of $s p^{3}$ hybridized carbon atoms. The bulk modulus of fcc structures is also large, though not as large as that for diamond. The calculated bulk moduli for the layered structures are quite small compared to three-dimensional polymers, which results from the weak van der Waals interaction between the planes. In particular, the elastic constants and the bulk modulus of the qgp structure (50 GPa in Table II) are about the same as for graphite (bulk modulus is $34 \mathrm{GPa}$ in Table II). Calculated values for graphite and diamond are in excellent agreement with experimental results. $^{33,38}$

The results of the electronic structure calculations for all the found polymer structures are summarized in Table I and figures in Ref. 3, where the density of states and band structure plots are presented. Metallic conductivity is observed for qgp, hcp, and $\mathrm{sc}_{\text {open }}$ structures. The $3 \mathrm{D}$ structures are semiconducting except $\mathrm{sc}_{\mathrm{open}}$, which is metallic. The band-gap values for semiconductor solids vary from 1.4 (for $\mathrm{bcm}_{\text {closed }}$ ) to $2.57 \mathrm{eV}$ (for $\mathrm{C}_{22} \mathrm{fcc}$ ). Our results are in good agreement with the calculations by $\mathrm{Chen}^{18}$ for the $\mathrm{bcm}_{\text {closed }}$ structure and by Miyamoto ${ }^{5}$ for the $\mathrm{sc}_{\text {open }}$ structure. The calculated band gap value for $\mathrm{C}_{22} \mathrm{fcc}$ is slightly larger compared to the value $2.47 \mathrm{eV}$ calculated in a previous study ${ }^{15}$ using LDA, which is known to underestimate the band gap. Band structure plots indicate a direct band gap for the $\mathrm{fcc}_{22}$ structure, while the others are indirect band gap semiconductors, see Fig. 3.

Let us now focus on the electronic properties of the qgp structure, which is the energetically most favorable polymer in our studies. This structure manifests metallic properties along the tube axis but no metallic conductivity in in-plane $(\Gamma-X)$ and perpendicular $(\mathrm{Y}-\Gamma)$ directions (see the band structure and electronic DOS shown in Ref. 35). In contrast to the electronic structure of graphite, there are two well-localized states in the $(\Gamma-\mathrm{X})$ and $(\mathrm{Y}-\Gamma)$ directions seen in the band gap. In order to excite an electron from the lowest of these localized states, which are fully occupied, to the next empty level one needs only 0.69 and $0.40 \mathrm{eV}$ in $(\Gamma-\mathrm{X})$ and $(\mathrm{Y}-\Gamma)$ directions, respectively. This electronic band property can possibly be used in applications that require photons with high frequency.

\section{Stability}

The stability of the considered polymers was studied using the Parrinello-Rahman molecular dynamics (MD) NPT technique implemented in the CPMD code. Both isotropic and anisotropic strains were considered. The performed molecular dynamics calculations showed that all the structures sustain pressures up to $20 \mathrm{GPa}$ at temperatures below $1000 \mathrm{~K}$. However, at higher pressures phase transformations can occur, especially when the pressure is rapidly increased. Structures that are energetically less favorable, like sc structures, undergo phase transformation much easier than fcc or qgp structures. For example, the qgp structure endures pressures up to $50 \mathrm{GPa}$ without any phase transformation, while $\mathrm{sc}_{\text {closed }}$ splits to graphitelike planes already at $30 \mathrm{GPa}$. The high-pressure phase for the qgp structure was found to be an amorphous-diamond phase.

\section{Vibrational properties}

In addition to the MD stability calculations, the nature of phonon vibrational modes can be used to study the stability of the structures. Absence of zero-mode components in the calculated phonon dispersion spectrum is strong evidence for the stability of a structure. A set of dispersion curves for selected structures is shown in Fig. 4. For the $\mathrm{C}_{22}$-fcc structure, the calculated dispersion relation is in good agreement with calculations by Spagnolatti et al. ${ }^{39}$ The phonon dispersion curves were calculated using finite differences and the ab initio perturbation theory approach implemented in the ABINIT $^{28}$ code with LDA approximation. In order to estimate the sensitivity of the calculated frequencies to the choice of the exchange-correlation approximation and the code used, the phonon modes were also calculated using both LDA and GGA approximations and VASP. In all tested cases, GGA gives approximately $50 \mathrm{~cm}^{-1}$ lower frequencies than LDA, which correlates with the known fact that LDA overestimates interatomic binding, whereas GGA underestimates it. With LDA, VASP gives the same results as ABINIT.

Raman spectroscopy provides another fingerprint by which a structure can be identified, since vibrational information is very sensitive to the spatial disposition of chemical bonds that is unique for each structure. We have also calculated Raman spectra for all the structures, which can be used by (a)

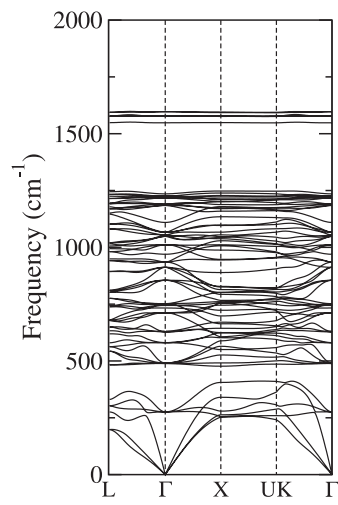

(c)

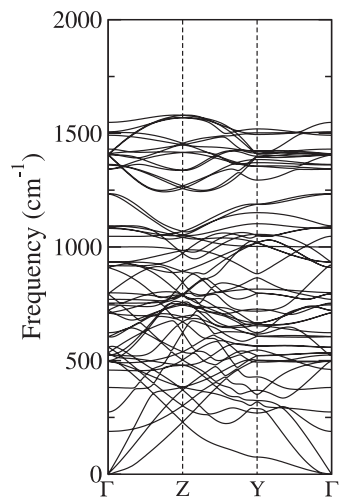

(b)

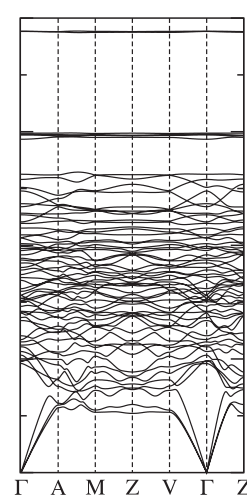

(d)

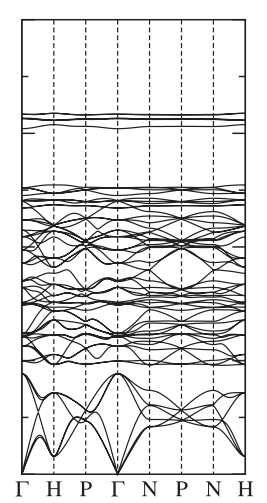

(c)

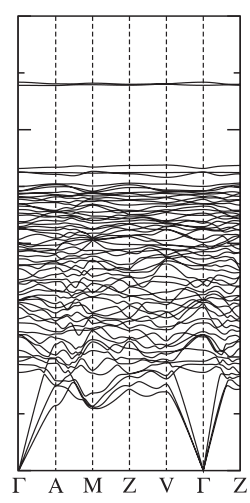

(e)

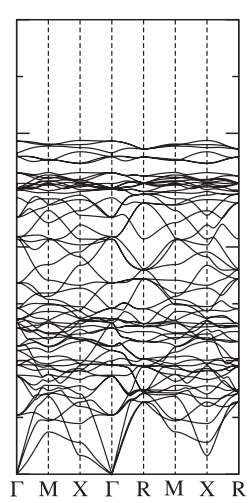

FIG. 4. Calculated dispersion relations for (a) fcc-22 (b) bcm closed, $_{\text {, }}$ (c) $b_{c m}$ open, (d) qgp (e) bcc, and (f) $\mathrm{sc}_{\text {open }}$ structures. 

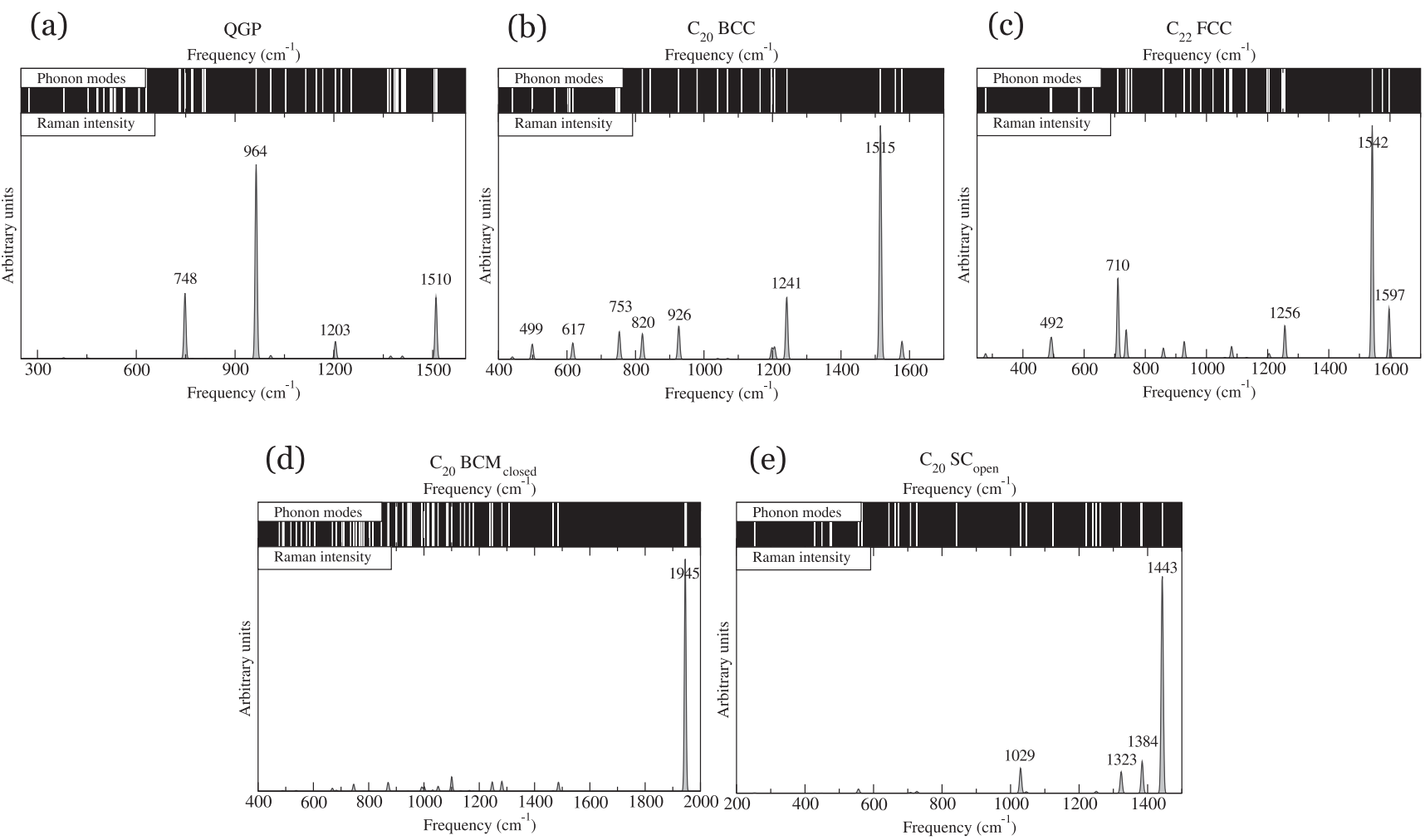

(e)
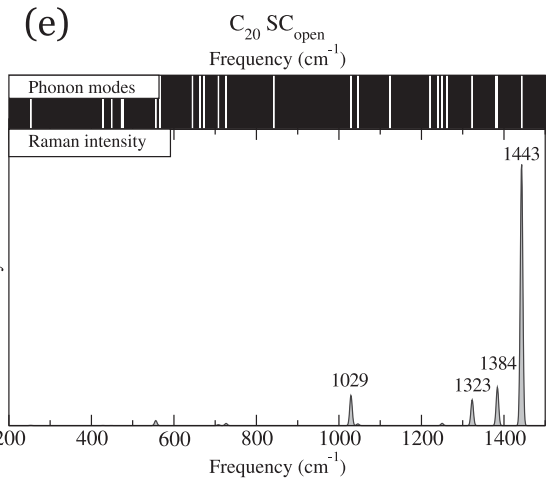

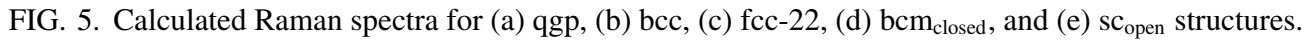

experimentalists for identification of structures synthetized in experiments. Raman intensities are calculated within the LDA approximation using ABINIT. Linear-response calculations give the Raman susceptibility tensor $R$, whose elements are derivatives of polarizability. The intensity of a phonon mode is proportional to the largest eigenvalue of $R^{*} R$. A mode is Raman active only if the motion occurs with a changing polarizability. At some distance $\Delta r$ away from the equilibrium structure the polarization $\alpha$ is given by

$$
\alpha \approx \alpha_{0}+\frac{\partial \alpha}{\partial r} \Delta r,
$$

where the term $\partial \alpha / \partial r$ is the change in polarizability with change in a nuclear position. If the derivate is zero, no Raman scattering occurs. In-depth computational details and implementation of the method in ABINIT code can be found in the review article by Baroni et al. ${ }^{40}$ and the articles by Gonze et al. ${ }^{41-43}$

So far, only one experimental Raman spectrum for the $\mathrm{C}_{20}$ solid structure has been measured. ${ }^{15}$ This spectrum is claimed to belong to the $\mathrm{C}_{22}$ structure, although the fit between experimental and computational data is not perfect. The calculated Raman spectra for solids studied in this work are presented in Fig. 5. For the $\mathrm{C}_{22}$ structure, the calculated Raman spectrum shows features similar to those calculated by Iqbal et al. ${ }^{15}$ The most dominant peak positions at 1561, 1506, 729, 697, and $488 \mathrm{~cm}^{-1}$ and their intensities calculated in Ref. 15 are in agreement with our calculations, see Fig. 5(c). At the same time, our calculations give a better agreement with experimental peak positions for the two main peaks located at 1585 and $1615 \mathrm{~cm}^{-1}$, though their intensities differ significantly from the measured ones.

In the qgp, $\mathrm{C}_{20}$ bcc, $\mathrm{C}_{20} \mathrm{sc}_{\text {open }}$, and $\mathrm{C}_{22}$ fcc spectra we can identify a strong $G$ band located near $1500 \mathrm{~cm}^{-1}$, which is split into several low-intensity peaks. This feature is similar to the $G$ band in graphene $\left(1580 \mathrm{~cm}^{-1}\right)$ and carbon nanotubes, where the $G$ band is split into many features around $1582 \mathrm{~cm}^{-1} \cdot 44$ In contrast to the other structures where the $G$ band has the highest Raman intensity, in qgp the Raman spectrum exhibits peaks at 748 and $964 \mathrm{~cm}^{-1}$ whith larger intensities. Also, the $\mathrm{C}_{20} \mathrm{bcm}_{\text {closed }}$ structure has a unique Raman signature at $1945 \mathrm{~cm}^{-1}$, which cannot be confused with the Raman active modes found in other structures. The main Raman peaks of qgp and $\mathrm{C}_{20}$ bcm closed are absent in other $\mathrm{C}_{20}$ structures and therefore can be used to identify unequivocally these structures in experimental spectra.

\section{CONCLUSIONS}

Several $\mathrm{C}_{20}$-based solid structures are proposed, based on first-principles density-functional calculations. The proposed structures are stable against external strain (at least up to $20 \mathrm{GPa}$ ) and high temperature (at least to $1000 \mathrm{~K}$ ). The elastic and electronic properties of the structures are calculated. It is shown, in particular, that $\mathrm{fcc}$, bcc, and $\mathrm{sc}_{\text {closed }}$ structures manifest the semiconducting properties with the band gap varying from 1.0 to $2.57 \mathrm{eV}$, while the layered qgp and hcp exhibit metallic conductivity in the in-plane directions. Phonon dispersion curves show the stability of the structures. Calculated Raman spectra can be used for structure identification in experimental studies. 


\section{ACKNOWLEDGMENTS}

This work was supported by the Academy of Finland through project SA120004 and in the framework of the Center of Excellence Program (2006-2011). We are grateful to Center of Scientific Computing (CSC), Espoo for generous computational resources. The pictures of the molecular structures were drawn using $\mathrm{VMD}^{45}$ visualization package. *vehvilainen@iki.fi

${ }^{1}$ Z. Yang, Y. Xia, and R. Mokaya, J. Am. Chem. Soc. 129, 1673 (2007).

${ }^{2}$ Y.-H. Kim, Y. Zhao, A. Williamson, M. J. Heben, and S. B. Zhang, Phys. Rev. Lett. 96, 016102 (2006).

${ }^{3}$ Y. Zhao, Y.-H. Kim, A. C. Dillon, M. J. Heben, and S. B. Zhang, Phys. Rev. Lett. 94, 155504 (2005).

${ }^{4}$ Y. Ren, T. Ng, and K. Liew., Carbon 44, 397 (2006).

${ }^{5}$ Y. Miyamoto and M. Saito, Phys. Rev. B 63, 161401 (2001).

${ }^{6}$ S.-H. Ke, H. U. Baranger, and W. Yang, Phys. Rev. Lett. 91, 116803 (2003).

${ }^{7}$ A. S. Alexandrov, A. M. Bratkovsky, and R. S. Williams, Phys. Rev. B 67, 075301 (2003).

${ }^{8}$ Y. Tabata and Y. Ikada, Pure Appl. Chem. 71, 2047 (1999).

${ }^{9}$ W. Noon, Y. Kong, and J. MA, Proc. Natl. Acad. Sci. USA 99, 6466 (2002).

${ }^{10}$ T. Kyotani, Bull. Chem. Soc. Jpn. 79, 1322 (2006).

${ }^{11}$ Z. Ma, T. Kyotani, and A. Tomita, Carbon 40, 2367 (2002).

${ }^{12}$ H. Kroto, J. Heath, S. O'Brien, and R. Smalley, Nature (London) 318, 162 (1985).

${ }^{13}$ S. Okada and A. Oshiyama, Phys. Rev. B 68, 235402 (2003).

${ }^{14}$ T. A. Beu, J. Onoe, and A. Hida, Phys. Rev. B 72, 155416 (2005).

${ }^{15}$ Z. Iqbal, Y. Zhand, H. Grebel, S. Vijayalakshmi, A. Lahamer, G. Benedek, M. Bernasconi, J. Cariboni, I. Spagnolatti, R. Sharma, R. F. J. Owens, M. E. Kozlov, K. V. Rao, and M. Muhammed, Eur. Phys. J. B 31, 509 (2003).

${ }^{16}$ I. Spagnolatti, M. Bernasconi, and G. Benedek, Europhys. Lett. 59, 572 (2002).

${ }^{17}$ Z. Wang, X. Ke, Z. Zhu, F. Zhu, M. Ruan, H. Chen, R. Huang, and L. Zheng, Phys. Lett. A 280, 351 (2001).

${ }^{18}$ Z. Chen, T. Heine, J. Jiao, A. Hirsch, W. Thiel, and P. Schleyer, Chem. Eur. J 10, 963 (2004).

${ }^{19}$ T. Vehviläinen, J Nanoscience Nanotechnology 9, 4360 (2009).

${ }^{20}$ S. Okada, Y. Miyamoto, and M. Saito, Phys. Rev. B 64, 245405 (2001).

${ }^{21}$ G. Kresse and J. Furthmüller, Phys. Rev. B 54, 11169 (1996).
${ }^{22}$ G. Kresse and J. Furthmüller, Comput. Mater. Sci. 6, 15 (1996).

${ }^{23}$ J. P. Perdew and Y. Wang, Phys. Rev. B 45, 13244 (1992).

${ }^{24}$ G. Kresse and D. Joubert, Phys. Rev. B 59, 1758 (1999).

${ }^{25}$ P. E. Blöchl, Phys. Rev. B 50, 17953 (1994).

${ }^{26}$ R. Car and M. Parrinello, Phys. Rev. Lett. 55, 2471 (1985).

${ }^{27}$ CPMD, Copyright IBM Corp 1990-2006, Copyright MPI für Festkörperforschung Stuttgart 1997-2001 (1997-2001).

${ }^{28}$ X. Gonze et al., Comput. Mater. Sci. 25, 478 (2002).

${ }^{29}$ M. Elstner, P. Hobza, T. Frauenheim, S. Suhai, and E. Kaxiras, J. Chem. Phys. 114, 5149 (2001).

${ }^{30}$ W. T. M. Mooij, F. B. van Duijneveldt, J. G. C. M. van Duijneveldtvan de Rijdt, and B. P. van Eijck, J. Phys. Chem. A 103, 9872 (1999).

${ }^{31}$ W. T. M. Mooij, B. P. van Eijck, and J. Kroon, J. Phys. Chem. A 103, 9883 (1999).

${ }^{32}$ R. Williams and D. Malhotra, Chem. Phys. 327, 54 (2006).

${ }^{33}$ A. A. Ahmadieh and H. A. Rafizadeh, Phys. Rev. B 7, 4527 (1973).

${ }^{34}$ H. Rydberg, M. Dion, N. Jacobson, E. Schröder, P. Hyldgaard, S. I. Simak, D. C. Langreth, and B. I. Lundqvist, Phys. Rev. Lett. 91, 126402 (2003)

${ }^{35}$ M. G. Ganchenkova, T. T. Vehviläinen, and R. M. Nieminen, Phys. Rev. B 78, 195421 (2008).

${ }^{36}$ P. Ravindran, L. Fast, A. Korzhavyi, and B. Johansson, J. Appl. Phys. 84, 4891 (1998).

${ }^{37}$ F. Murnaghan, Proc. Natl. Acad. Sci. USA 30, 244 (1944).

${ }^{38}$ H. McSkimi and P. Andreatch, J. Appl. Phys. 43, 2944 (1972).

${ }^{39}$ I. Spagnolatti, A. Mussi, M. Bernasconi, and G. Benedek, Eur. Phys. J. B 37, 143 (2003).

${ }^{40}$ S. Baroni, S. de Gironcoli, A. Dal Corso, and P. Giannozzi, Rev. Mod. Phys. 73, 515 (2001).

${ }^{41}$ X. Gonze and J.-P. Vigneron, Phys. Rev. B 39, 13120 (1989).

${ }^{42}$ X. Gonze, Phys. Rev. A 52, 1096 (1995).

${ }^{43}$ X. Gonze and C. Lee, Phys. Rev. B 55, 10355 (1997).

${ }^{44}$ M. Dresselhaus, G. Dresselhaus, R. Saito, and A. Jorio, Phys. Rep. 409, 47 (2005).

${ }^{45}$ W. Humphrey, A. Dalke, and K. Schulten, J. Mol. Graphics 14, 33 (1996). 